\title{
In Vitro Polarized Resonance Raman Study of N719 and N719-TBP in Dye Sensitized Solar Cells
}

\author{
Søren Hassing ${ }^{1, *}$, Kit D. Jernshøj ${ }^{1}$, Phuong Tuyet Nguyen ${ }^{2}$ and Torben Lund ${ }^{3}$ \\ ${ }^{1}$ Department of Chemical Engineering, Biotechnology and Environmental Technology, Faculty of Engineering, \\ University of Southern Denmark, Campusvej 55, DK-5230 Odense M, Denmark \\ ${ }^{2}$ Chemistry Department, University of Science, Vietnam National University Ho Chi Minh City, Vietnam \\ ${ }^{3}$ Department of Science and Environment, Roskilde University, 4000 Roskilde, Denmark
}

\begin{abstract}
The working efficiency of dye-sensitized solar cells (DSCs) depends on the long-term stability of the dye itself and on the microscopic structure of the dye-semiconductor interface. Previous experimental studies of DSCs based on ruthenium dye with bipyridine ligands (N719) adsorbed to the $\mathrm{TiO}_{2}$ substrate applied FTIR, un-polarized Raman (RS) and un-polarized resonance Raman (RRS) spectroscopy. In the un-polarized RRS studies of $\mathrm{N}_{119 / T i O}-\mathrm{DSCs}_{2}$ the discussion of the adsorption of N719 was based on the rather weak carbonyl or carboxyl group stretching vibrations and on minor spectral changes of overlapping Raman modes, whereas conclusions about the dye-stability was based on the observation that fresh and aged DSCs had almost identical RRS spectra.

In the present paper we address the problems mentioned above, by utilizing the unique property of Raman scattering that the polarization of the scattered light is generally different from the polarization of the laser light. When the excitation is chosen within the visible absorption band of N719 only the skeleton ring-modes in N719 are enhanced and are observed as the most intense bands in the RRS spectra. We demonstrate by experimental results on $\mathrm{N}_{119} / \mathrm{TiO}_{2}-\mathrm{DSCs}$ that by combining an analysis of the wave number dependent polarization of these modes with the small shifts observed in the visible absorption spectra of adsorbed, non-adsorbed molecules and degradation products new and more reliable information about dye stability and about the adsorption of the dye on $\mathrm{TiO}_{2}$ can be obtained. Furthermore it is found that the polarization fluorescence anisotropy is very different for adsorbed and non-adsorbed dye molecules. This information is automatically obtained when processing the Raman data. The conclusion is that if the polarization properties of the resonance Raman spectra are analyzed instead of just analyzing the minute spectral changes of the (weaker) Raman bands the potential of RRS as an on-site tool for investigation of DSCs can be greatly improved.
\end{abstract}

Keywords: Dye sensitized solar cell, Polarized Raman, polarized Fluorescence.

\section{INTRODUCTION}

Dye-sensitized solar cells (DSCs) have attracted much attention since Grätzel and coworkers proposed and demonstrated the applicability of using a dye as a sensitizer in converting sunlight into electricity [1-4]. The low production costs combined with the flexibility of the DSCs seem to offer attractive new possibilities with respect to integration of the DSCs into building components such as power generating windows and various façade applications. To develop the DSCs to be an attractive and real alternative to the more common silicon based solar cells, the DSC's must be developed to respond properly to the typical operation conditions and to possess a high efficiency in the energy conversion process. With respect to operation conditions the thermal appliance and long-term stability are of special importance. Since the dye is one of the key components of a DSC device, the thermal stabilityof the DSC is directly linked to the long-term stability of thedye. In addition to this the working

*Address correspondence to this author at the Department of Chemical Engineering, Biotechnology and Environmental Technology, Faculty of Engineering, University of Southern Denmark, Campusvej 55, DK-5230 Odense M, Denmark; Tel: +4565507414; Fax: +4565507384;

E-mail: sh@kbm.sdu.dk efficiency of the DSCs depends on the microscopic structure of the dye-semiconductor interface, which influences the chemical binding of the dye to the surface, which is important for the injection of electrons from the excited dye to the semiconductor. Finally the stability of the dye depends on the chemical composition of the electrolyte added to the dye and the reactivity of the additives towards the dye, when heated.

There is need for having a sensitive and nondestructive measuring technique for monitoring the dye stability, when the DSC is exposed to the typical working conditions. It is necessary to be able to measure on-site whether or not the dye is degraded upon illumination and heating. Since in the degradation process substitution products are formed the technique must be molecule specific. Likewise it is important for further development of the DSCs to increase the amount of information that may be extracted about the adsorption - desorption of the selected dye to the semiconductor surface.

Raman spectroscopy is attractive as an experimental technique for on-site investigations of dye sensitized solar cells. It is a fast, non-destructive and 
molecule specific technique, which requires no special sample preparation and the excitation and collection of the Raman scattered light can be performed through the cover glass of the DSCs. Accordingly both Raman and resonance Raman spectroscopy have been applied in numerous investigations of e.g. the stability of the DSCs $[5,6]$.

A Raman spectrum contains the unique and highly resolved vibrational signature of the molecule and it is obtained by illuminating the sample with polarized laser light and monitoring the backscattered light as a function of wave number. In RRS the wave number of the laser is chosen close to an electronic absorption in the molecule with the consequence that only vibrations associated with this electronic transition are enhanced in the Raman spectrum. In all the Raman investigations of DSCs found in the literature [7-11] only the Raman spectrum itself has been monitored and analyzed. Thus the technique has been applied in a similar way as when IR, FTIR and ATR-IR spectroscopy are applied $[9,12,13]$.

A unique property of the Raman process is that the polarization of the scattered light, obtained from both oriented molecules as well as from solutions and powders, will generally be different from the polarization of the incident laser light. The change of the polarization will depend on the nature of the vibrational modes contributing to the scattering and the polarization change will depend on the wave number of the laser light, when the excitation is performed close to resonance with an electronic transition in the molecule [14].

In the present paper it is demonstrated that the reliability, the kind and amount of information about the
DSCs can be increased considerably by analyzing the change of the polarization of the Raman scattered light relative to the polarization of the incident laser light. It is also demonstrated that the polarization change of the emitted fluorescence is very different for adsorbed and desorbed dye molecules.

Since the visible absorption spectra of the dye, the degraded dye and the dye desorbed from the semiconductor exhibit small shifts in wave numbers i.e. 10-30 nm (see Figure 10 and $[15,16]$ ) and since the polarization properties of the various vibrational modes depends on the particular excitation wave number applied, it follows that the polarization of the resonance enhanced modes i.e. the skeleton vibrations of the chromophore (dye) will change. Recently we have applied this idea of combining a shift in the visible absorption with the polarization properties of the Raman modes by investigating solutions of the dyecomplex $\mathrm{N} 719$ and its main degradation product $\left[\mathrm{Ru}(\mathrm{LH})_{2}(\mathrm{NCS})(4-\right.$ tert-butylpyridine $\left.)\right]\left[\mathrm{N}(\mathrm{Bu})_{4}\right], \quad$ (N719TBP for short) [15]. The investigations of DSC samples of $\mathrm{N} 719 / \mathrm{TiO}_{2}$ performed in the following forms the next natural step in demonstrating that polarized resonance Raman scattering has a larger potential than unpolarized resonance Raman scattering for being developed into a more optimized tool for on-site investigations of DSCs also because the method discussed here has general applicability.

\section{MATERIALS AND METHODS}

\section{Materials}

The bis(tetrabutylammonium) salt of the complex cis-bis(isothiocyanato)bis(2,2'-bipyridyl-4,4'-dicarboxylato)ruthenium(II), with the trade name Ruthenium 535-

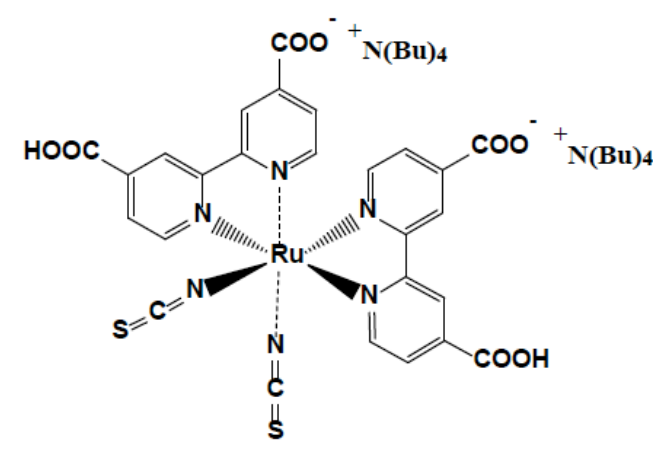

N719

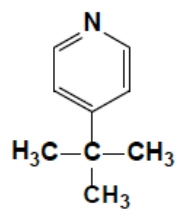

TBP

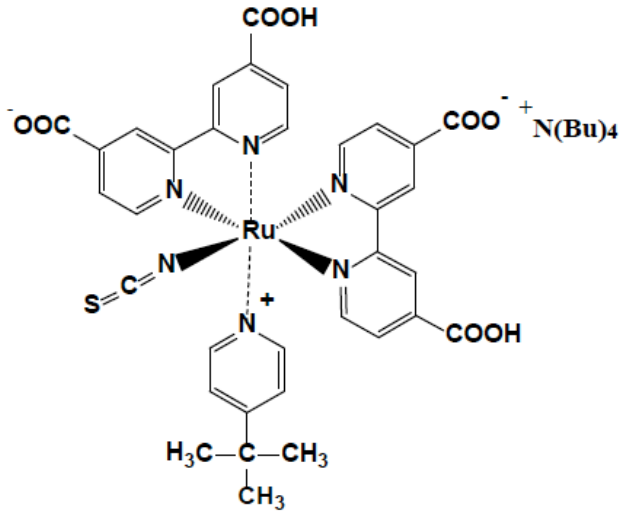

N719-TBP

Figure 1: Chemical structure of the dyes N719, N719-TBP and of the additive TBP. 
bis TBA and referred to as N719, was obtained from Solaronix, Aubonne, Switzerland. 4-tert-butylpyridin (TBP) and 3-methoxy-propionitrile (3-MPN) were purchased from Sigma-Aldrich and used as received. The substitution product N719-TBP (see [15]) was synthesized according to the protocol of Nguyen et al. [17].

The chemical structures of the dyes N719 and N719-TBP and of the additive TBP are shown in Figure 1.

\section{Fabrication of DSCs}

The cells were fabricated with Solaronix DSC kit containing ready-to-use transparent titania photo anodes and platinium counter electrodes together with gaskets, sealing sand caps (Solaronix catalog number 7499). The titania kit electrodes have a surface area of $0.36 \mathrm{~cm}^{2}$ and consist of three layers of active dye absorption titania, with a total thickness about $10 \mu \mathrm{m}$. The kit electrodes were re-sintered at $450{ }^{\circ} \mathrm{C}$ for $15 \mathrm{~min}$ prior before use. Titania kit electrodes were dye soaked for 12 hours in 7 dye different baths containing various concentrations of the two dyes N719 and N719-TBP dissolved in methanol and with [N719]+[N719-TBP] = $0.50 \mathrm{mM}$. The concentration of N719-TBP increased from $0,5,10,20,30$, and 40 to $100 \%$. The dyed photo anodes were rinsed with methanol and assembled into a cell using the Solaronix kit polymeric sealant. Finally, the cell was filled with an electrolyte comprised of a mixture of $0.05 \mathrm{M} \mathrm{I}_{2}, 0.1 \mathrm{M} \mathrm{Lil}, 0.6 \mathrm{M}$ tetrabutylammoniumiodide and $0.5 \mathrm{M}$ 4-tert-butylpyridine in 3MPN and the filling hole were sealed.

After dye-soaking one titania kit electrode from each of the seven dye baths were selected and rinsed with methanol. The dye was desorbed from the $\mathrm{TiO}_{2}$ surface by treatment with $0.1 \mathrm{M} \mathrm{NaOH}(1 \mathrm{ml})$. The extract was acidified by $20 \mu \mathrm{l}$ of formic acid and analysed by HPLCUV/Vis according to a previously described analysis protocol [18]. A photo of a representative example of the DSC samples used in the present study is shown in Figure 2. The thin film of the dye, $\mathrm{TiO}_{2}$ and electrolyte is placed between two cover glass with the thickness of $2 \mathrm{~mm}$.

\section{Methods}

The polarized resonance Raman spectra were collected by using a fiber coupled $180^{\circ}$ Raman microscope. The DSC samples, as the one shown Figure 2, are placed under the microscope and the excitation and collection of the Raman scattered light

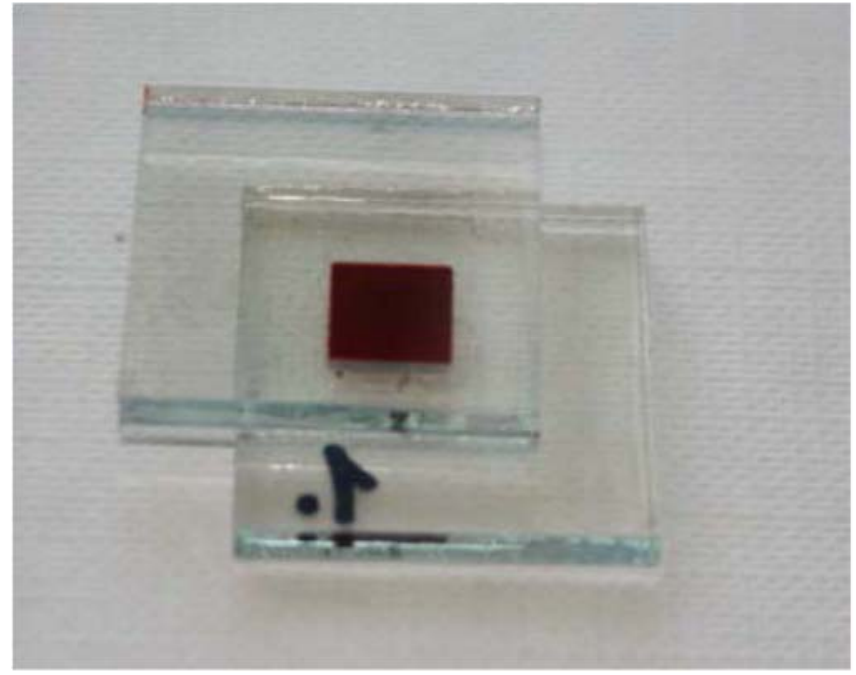

Figure 2: Photo of a representative DSC sample N719 / $\mathrm{TiO}_{2}$ fabricated as described in text. The Raman measurements are performed through the $2 \mathrm{~mm}$ thick cover glass by focusing the laser beam on to the red square containing the thin film of the dye, $\mathrm{TiO}_{2}$ and the electrolyte and collecting the backscattered Raman light (see text).

are performed through the $2 \mathrm{~mm}$ thick cover glass. The direction of the laser beam is approximately perpendicular to the glass surface. The Raman microscope consists of a modified Olympus BX60F5 microscope, a Spectra Pro 2500i spectrograph from Acton equipped with a 1200 lines/mm grating and a cooled CCD detector from Princeton Instruments, model Acton PIXIS. The laser excitation was provided by a polarized $532 \mathrm{~nm}$ cw laser (Ventus LP 532) combined with free-space optics to ensure that the polarization on the sample is well defined. Tominimize degradation of the dye as well as excess heating the laser power and the integration times were kept as low as possible (less than $5 \mathrm{~mW}$ and $30 \mathrm{sec}$.). The collection optics was optimized by using a dichroic mirror instead of a broadband beam splitter. A $10 \mathrm{x}$ objective with a 0.30 numerical aperture was used to focus the laser beam onto the sample and to collect the backscattered light. The spot size of the laser on the sample was approximately $5 \mu \mathrm{m}$. The measured perpendicular and parallel polarized scattered light, $I_{R, p e r}$ and $I_{R, p a r}$, were coupled into the spectrometer via a multimode fiber, where parallel and perpendicular mean that the polarization is either parallel or perpendicular to the polarization of the laser light. The depolarization ratio (DPR) of each Raman band is defined as the ratio $I_{R, p e r} / I_{R, p a r}$. As shown in reference [15] the laser influence on the sample can be minimized by measuring the polarized resonance Raman spectra in the following order in time: (1): a perpendicularly polarized spectrum $\left(I_{1, \text { per }}\right)$, (2): a parallel polarizedspectrum $\left(I_{2, p a r}\right), \quad(3)$ : a perpendicularly polarized spectrum $\left(I_{3, p e r}\right)$ and (4): a 
parallel polarized spectrum $\left(I_{4, p a r}\right)$ and then calculating improved DPR values in the following way:

$D P R=\frac{1}{2}\left(\frac{\left\langle I_{1,3, \text { per }}\right\rangle}{I_{2, \text { par }}}+\frac{I_{3, \text { per }}}{\left\langle I_{2,4, \text { par }}\right\rangle}\right)$

where $\left\langle I_{1,3, p e r}\right\rangle$ is the average of the spectra $I_{1, p e r}$ and $I_{3, \text { per }}$ and $\left\langle I_{2,4, p a r}\right\rangle$ is the average of $I_{2, p a r}$ and $I_{4, p a r}$. Before the measurements on the DSCs were performed the parallel and perpendicularly polarized intensities of the Raman scattered light were corrected for the difference in instrumental sensitivity by using the measured parallel and perpendicular components of the asymmetric vibrations in benzene (848.9 and $\left.1178 \mathrm{~cm}^{-1}\right)$ and cyclohexane $(1029,1267$ and $\left.1443 \mathrm{~cm}^{-1}\right)$. Besides, the spectrometer was wave number calibrated relative to the symmetric stretch vibration at $992 \mathrm{~cm}^{-1}$ of the benzene standard.

In fluorescence spectroscopy the change in polarization is conventionally described by the anisotropy $r_{f}$ defined as [19],

$r_{f}=\left(\frac{I_{F, p a r}-I_{F, p e r}}{I_{F, p a r}+2 I_{F, p e r}}\right)$

where $I_{F, p a r}$ and $I_{F, p e r}$ are the intensities of the emitted fluorescence with polarization parallel and perpendicular to the polarization of the exciting light, respectively.

The visible absorption spectra for the DSC-samples were obtained by measuring the reflection and transmission coefficients and applying energy conservation. The measurements were performed with a Li-COR LI-1800 spectrometer equipped with an integrating sphere (for further details, see [16]). The visible absorption spectra for the dye solutions N719 and N719-TBP in methanol were obtained by using a Lambda 900 spectrometer (Perkin Elmer) and a precision quartz cuvette with $1 \mathrm{~mm}$ path length. For comparison the spectra are collected in Figure $\mathbf{1 0 .}$ Since we are only interested in comparing the wave numbers for maximum absorption, arbitrary units have been used for both the absorptance of the DSCs and the absorbance of the solutions.

\section{RESULTS AND DISCUSSION}

We have obtained un-polarized and polarized resonance Raman spectra in the region 200 to $2800 \mathrm{~cm}^{-1}$ for the solar cell samples $D S C_{100 / 0}, D S C_{80 / 20}$, $D S C_{50 / 50}, D C S_{20 / 80}$ and $D C S_{0 / 100}$, where the first index refers to the relative amount of N719 and the second to N719-TBP. The un-polarized RRS spectra were obtained as the sum of the perpendicular and parallel polarized spectra. As representative examples the

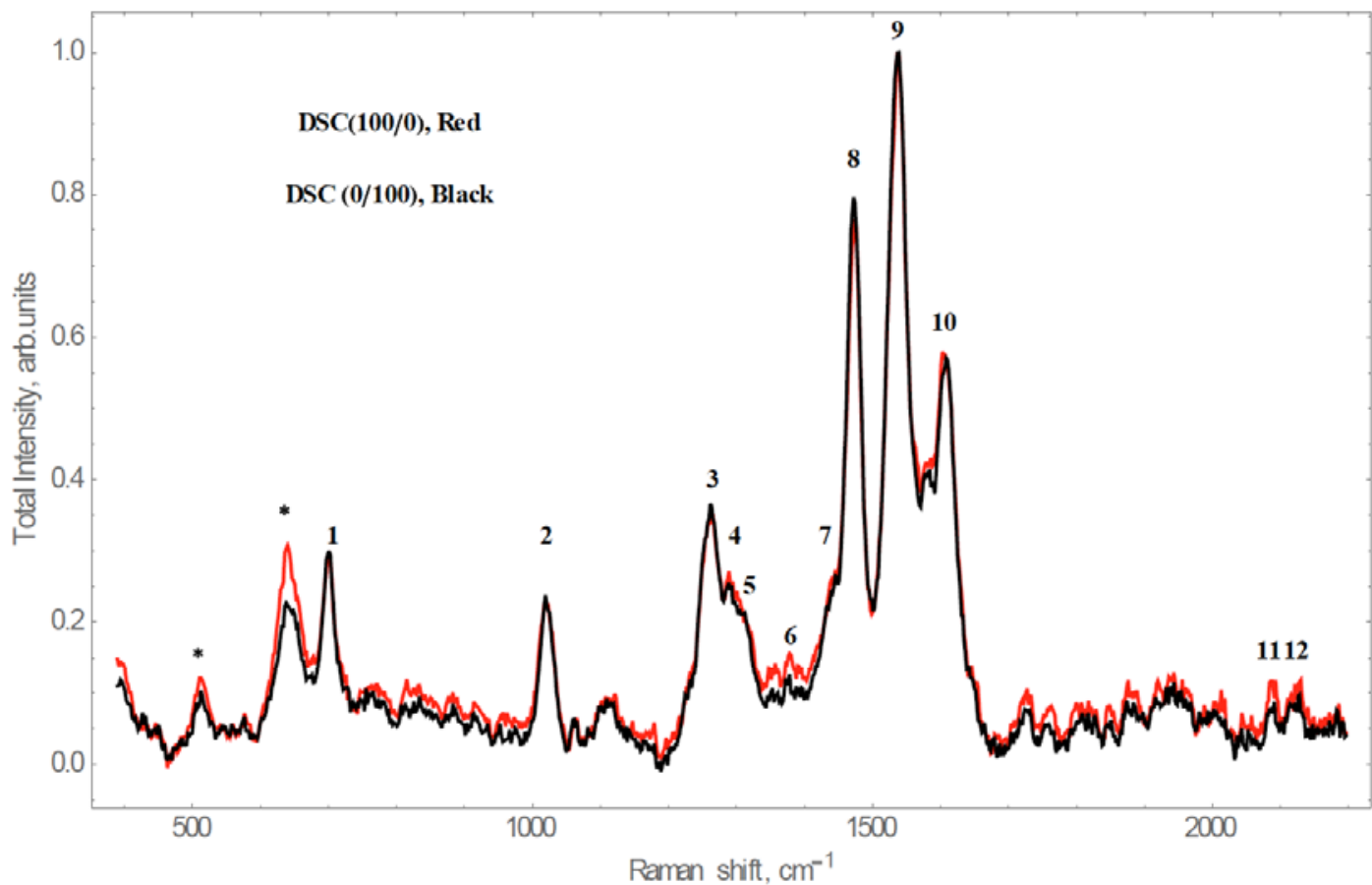

Figure 3: Fluorescence corrected un-polarized resonance Raman spectra for $D S C_{100 / 0}$ (red) and $D S C_{0 / 100}$ (black). Each spectrum has been scaled relative to the Raman band at $1536 \mathrm{~cm}^{-1}$, and the most prominent bands have been numbered $1-12$. $\mathrm{TiO}_{2}$ bands are marked *. 
fluorescence corrected un-polarized spectra for $D C S_{100 / 0}$ and $D C S_{0 / 100}$ are shown in Figure 3. The Raman bands are numbered 1 to 12 and the assignment of the bands is based on the assignment in reference [7]. For comparison purposes the Raman bands in each spectrum have been scaled relative to the intensity of the strongest Raman band (band 9) at $1536 \mathrm{~cm}^{-1}$ We have re-measured the polarized RRS spectra on solutions of N719 and N719-TBP in methanol and performed the signal processing as outlined below. Within the experimental uncertainty the calculated DPR values for these solutions confirmed the results given in [15].

\section{Signal Processing}

Before the results are discussed, a few words about the signal processing. Since each DPR value is calculated as a ratio between two band intensities the signal processing of the data must be carried out with great care in order to obtain reliable values. The signal processing of polarized Raman data, in cases where overlapping Raman bands and fluorescence are present, may be rather time-consuming and not trivial, although simple in principle. With respect to removal of fluorescence, various automatized methods can be found in the literature e.g. the wavelet based method developed in the programming language $R$ [20]. As emphasized in [20] the method has the advantage of being independent of the experience of the spectroscopist. For our data, however, we concluded, after having tried this method, that the most optimized and consistent result was obtained by fitting the fluorescence background in the entire spectral range with a $6^{\text {th }}$ order polynomial. Thus, the signal processing of the perpendicularly and parallel polarized RRS data was performed as follows: The raw data was corrected for the difference in sensitivity of the experimental setup towards the two polarizations as previously mentioned. The Raman signal was separated from the fluorescence background, by first removing all Raman bands from the data (this step is crucial) and fitting a $6^{\text {th }}$ order polynomial to the remaining fluorescence signal. A comparison of the spectra obtained from the DSCs with different ratio between dye and degradation product showed that the position of the Raman bands exhibits only very small wave number shifts. When identical Raman bands in the different spectra were removed in the fitting of the fluorescence the same spectral windows were therefore applied, by which the introduction of errors was minimized. For each polarization the fluorescence corrected Raman spectra were obtained by subtracting the fitted fluorescence background from the total signal and in the calculation of the DPR values the Raman intensities were represented by the band areas, which were determined by fitting the data with a Gaussian line shape. The Gaussian line shape was chosen, since this line shape closely resembles the delta function response of our spectrometer. To obtain a larger $\mathrm{S} / \mathrm{N}$ ratio and thereby improve the data processing, the wave numbers and the bandwidths of the Raman modes were obtained by fitting the sum of the parallel and perpendicularly polarized spectra (i.e. the un-polarized spectra) with the Gaussian line shape. The values of the wave numbers and the bandwidths were then used in the

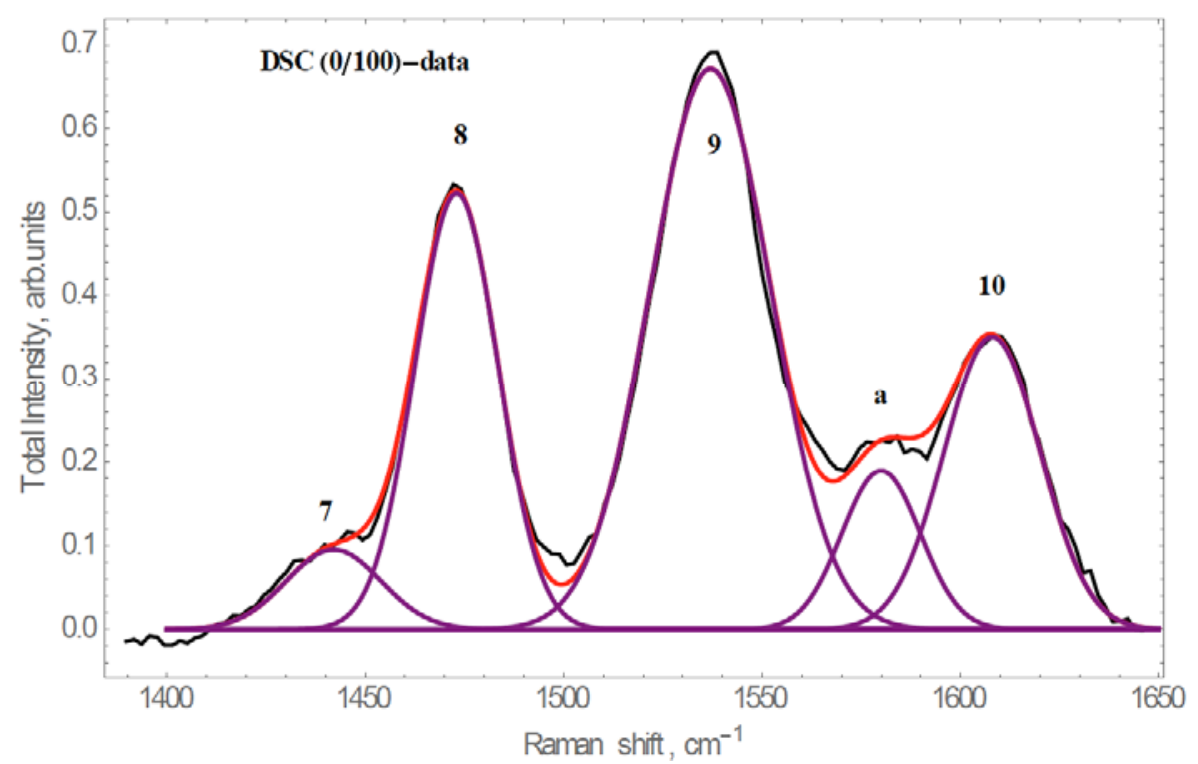

Figure 4: Best fit (red) of un-polarized Raman data for $D S C_{0 / 1000}$ (black) and decomposition in Gaussian components (purple). Band a at $1580 \mathrm{~cm}^{-1}$ has been not assigned. 
fitting of the parallel and perpendicularly polarized spectra. When overlapping bands are present in the spectra e.g. in the spectral ranges $1200-1350 \mathrm{~cm}^{-1}$ and $1430-1650 \mathrm{~cm}^{-1}$ the fitting procedure was carried out with a superposition of Gaussian functions. A representative example is shown in Figure 4.

\section{Discussion of the Un-Polarized RRS Spectra}

As seen from the Figure 3 our results show that the un-polarized RRS spectra for the cells $D S C_{100 / 0}$ and $D S C_{100 / 0}$ (where one should expect the largest difference) are nearly identical with respect to all the well-defined and prominent spectral features in the measured spectral region. The most striking difference appears to be a change in intensity of the $639 \mathrm{~cm}^{-1}$ band of the $\mathrm{E}_{\mathrm{g}}$ mode in $\mathrm{TiO}_{2}$ substrate. From analysis of the data small wave number shifts are found in the $D S C_{0 / 100}$ spectrum for the bands $2\left(-2 \mathrm{~cm}^{-1}\right), 3\left(-2 \mathrm{~cm}^{-1}\right)$, $4\left(+2 \mathrm{~cm}^{-1}\right), 8\left(-2 \mathrm{~cm}^{-1}\right)$ and $10\left(-5 \mathrm{~cm}^{-1}\right)$ where the numbers in the braketts are the shifts relative to the bands in the spectrum of $D S C_{100 / 0}$. We also determined the wave number shifts for the bands in the RRS spectrum of $D S C_{50 / 50}$ and found that most shifts were either zero or less than those obtained for $D S C_{0 / 100}$. Besides minor spectral changes in the region $1200-$ $1400 \mathrm{~cm}^{-1}$ are observed in both samples. Finally Figure 3 shows two weak bands, marked 11 and 12, at 2089 $\mathrm{cm}^{-1}$ and $2124 \mathrm{~cm}^{-1}$. In the literature $[5,7,9,10]$ the presence of spectral activity, both in Raman and IR, around $2100 \mathrm{~cm}^{-1}$ in DCSs with $\mathrm{N} 719 / \mathrm{TiO}_{2}$ was interpreted as involving the $\mathrm{CN}$-stretching mode of the thiocyanate group. Since at elevated temperatures (e.g. $85^{\circ} \mathrm{C}$ ) the additive 4-TBP may substitute one or two thiocyanate groups in N719, a spectral change in this area is expected to be sensitive to the degradation of $\mathrm{N} 719 / \mathrm{TiO}_{2}$ cell. A detailed discussion of the mechanism of thiocyanate ligand exchange can be found in [7]. In [9] abroad and weak band at $2090 \mathrm{~cm}^{-1}$ is reported in the RRS spectrum obtained with $532 \mathrm{~nm}$ excitation of $\mathrm{N} 719$ adsorbed to $\mathrm{TiO}_{2}$. In [5] $514 \mathrm{~nm}$ excitation was applied and a weak and broad band was observed in both fresh and aged $\mathrm{N} 719 / \mathrm{TiO}_{2}$ DSCs. They decompose numerically the band into two bands at 2108 and $2128 \mathrm{~cm}^{-1}$ and interpret the latter as the $\mathrm{CN}$-stretching mode. This agrees well with the band 12 in Figure 3. In [5] it is concluded that the stable intensity of the band at $2128 \mathrm{~cm}^{-1}$ for the fresh and aged DSCs indicates that there is no loss or substitution of the thiocyanate ligand upon aging. However, the data presented in Figure 7 of [5] shows that the intensity of the $\mathrm{CN}$-stretching band is actually increased approximately $20 \%$ in the aged DSC. Due to the apparent $20 \%$ increase of the intensity this number seems to represent the uncertainty of the stability argument. We have fitted the bands 11 and 12 in Figure 3 with a superposition of Gaussian functions and estimated that the intensity of the $\mathrm{CN}$-stretching band (band 12) in the sample $D S C_{0 / 100}$ is decreased to approximately $75 \%$ of the intensity of $D S C_{100 / 0}$. Since all N719 molecules have been substituted with N719TBP in the sample $D S C_{0 / 100}$ the observed decrease in intensity defines the order of magnitude of the maximum change, one may expect in the estimation of the degradation of the DSC. However, due to the small $\mathrm{S} / \mathrm{N}$ ratio for this band the estimation of the intensity becomes sensitive to the uncertainty in determining the fluorescence background. Besides the calculation of the band intensity requires as seen deconvolution of a signal with low $\mathrm{S} / \mathrm{N}$ ratio, which may also increase the uncertainty of the estimated intensity.

In order to elucidate this further we have also estimated the intensity of the $\mathrm{CN}$-stretching band for $D S C_{80 / 20}, D S C_{50 / 50}, D S C_{20 / 80}$ and performed a linear, least square fit to all five dataset. This gave the following values of the ratio between the intensity of the $\mathrm{CN}$-band in each sample and the intensity of the band in the sample $D S C_{100 / 0}, D S C_{0 / 100}: D S C_{0 / 100}: 1.00$, $D S C_{80 / 20}: 0.93, \quad D S C_{50 / 50}: 0.83, \quad D S C_{20 / 80}: 0.74$ and $D S C_{100 / 0}: 0.68$ with the standard deviation $\sigma=0.13$. It follows that the estimation of the relative contents of N719-TBP in the DSCs by monitoring the change of the un-polarized Raman intensity of the $\mathrm{CN}$-stretching band will be encumbered with large uncertainty. It is seen that the estimated standard deviation corresponds well with the $20 \%$ uncertainty observed for this band in [5].

The first important step in the working cycle of the DSCs is the excitation of the dye by visible light. The second step is the injection of electrons from the excited dye into the $\mathrm{TiO}_{2}$ substrate. The efficiency of the charge injection, but also the DSC stability, depends strongly on the kind of binding between the dye and the $\mathrm{TiO}_{2}$ substrate. In general it is accepted that the binding between the dye complex and the $\mathrm{TiO}_{2}$ surface is established via the carboxy ligands and that the coordination these occurs via bidentate bonds [9 and ref. therein]. In these studies un-polarized, resonance and non-resonance Raman spectroscopy as well as IR has been applied to DSCs and the results have been compared with results obtained for dye solutions and powders. The discussion includes the observed wave number shift of the broad and weak $\mathrm{CN}$-band near $2090 \mathrm{~cm}^{-1}$ relative to its position in the 


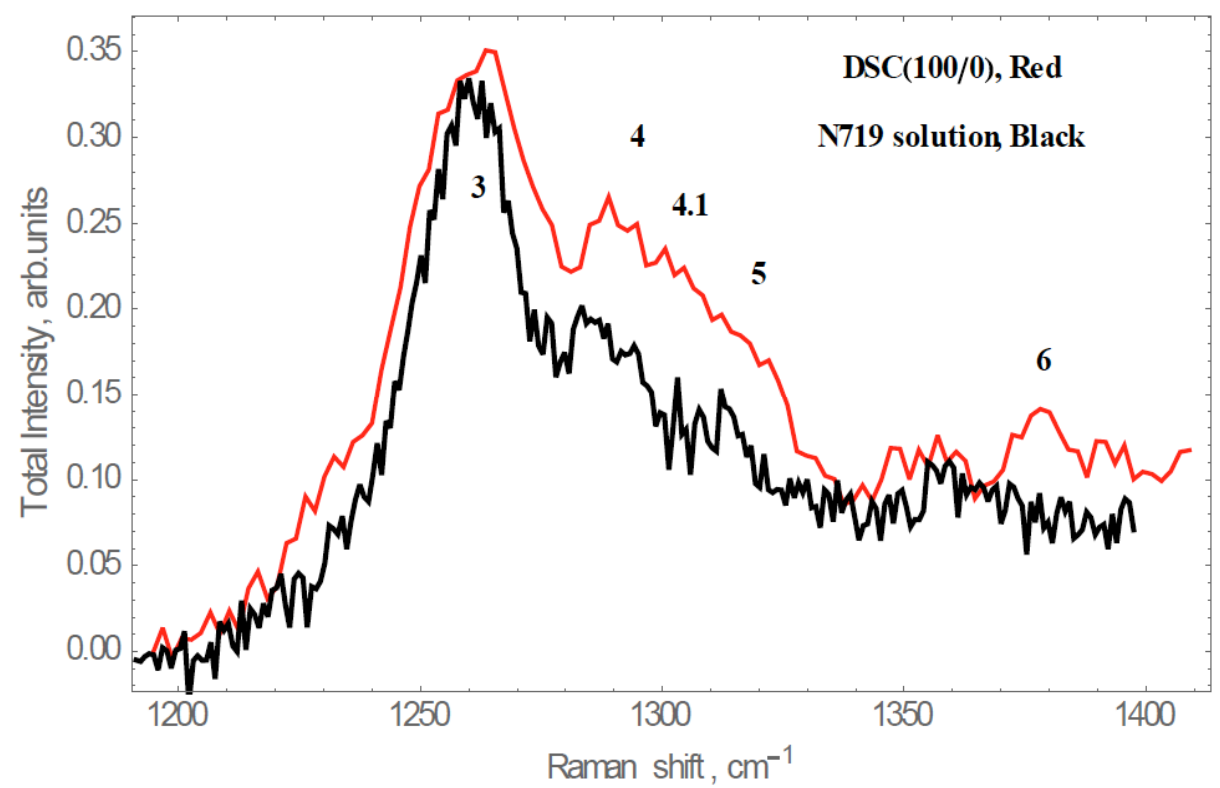

Figure 5: Un-polarized RRS spectra of $D S C_{100 / 0}$ and N719 in solution. Both spectra contains the bands 3,4 and 5 at 1264,1287 and $1312 \mathrm{~cm}^{-1}$. In the spectrum of the DSC we see either a broadening of the 1287 and 1312 bands or a new band (band 4.1 ) at $1304 \mathrm{~cm}^{-1}$. The largest is difference between the two spectra is the appearance of a new weak band at $1378 \mathrm{~cm}^{-1}(\mathrm{band} 6)$ in the DSC spectrum. Band 6 is assigned to the symmetric COO vibration [9].

RRS spectrum of N719 powder. In addition the discussion involves the spectral changes observed in the region $1200-1400 \mathrm{~cm}^{-1}$. The bands in this region are interpreted as being due to the totally symmetric stretching vibrations of the bipyridine ligand. As mentioned in [9] one difficulty in applying RRS in the investigation of the adsorption - desorption process is that due the resonance enhancement both adsorbed and non-adsorbed molecules may contribute to the resulting Raman spectra and since the spectral changes of the un-polarized spectra are small it will be nearly impossible to determine e.g. the ratio between adsorbed and non-adsorbed molecules. To illustrate the magnitude of the spectral changes which are typically achieved we show in Figure 5 the un-polarized RRS spectra of $D S C_{100 / 0}$ (red) and of N719 in solution (black] in this region.

It follows from the figure that the N719 spectrum contains 3 bands at 1264,1287 and $1312 \mathrm{~cm}^{-1}$ in agreement with [9], while in the spectrum of the DSC we see again these three bands and either a broadening of the 1287 and 1312 bands or a new band (band 4.1) at $1304 \mathrm{~cm}^{-1}$. The largest difference is the appearance of a new weak band at $1378 \mathrm{~cm}^{-1}$ (band 6) in the DSC spectrum. In ref [9] this band (reported at $1372 \mathrm{~cm}^{-1}$ ) is assigned to the symmetric COO vibration and the appearance of this band in the DSC spectrum is used to argue for the bidentate-coordination of the carboxy ligands to the $\mathrm{TiO}_{2}$ surface. Although we agree on the conclusion it follows from Figure 5 that although spectral changes are observed in this region these are not very large and only related to the bands with lowest intensity.

\section{Polarized RRS and Polarization Change of the Fluorescent Emission}

As already mentioned, we have in [15] taken a first step in the investigation of the possibilities for applying polarized RRS instead of un-polarized RRS in the studies of the DSCs. In [15] the solutions of the dyes N719 and N719-TBP in methanol were studied. It was found that although the un-polarized RRS spectra for these solutions were practically identical, the DPR values obtained for almost all strong Raman bands were significantly changed. But most importantly, the difference in the polarized fluorescence anisotropy $r_{f}$ for solutions containing either N719 or N719-TBP was so large that it should be easy to detect the presence of the degraded molecule N719-TBP in a solution of N719. For the solution of N719 the anisotropy was found to be $r_{f} \cong 0.2$, while for the solution of the substituted dye N719-TBP the anisotropy was increased with a factor $2, r_{f} \cong 0.4$. In both cases $r_{f}$ was found to be nearly independent of wave number. Although none of the molecules have any exact symmetry the significantly larger fluorescence anisotropy obtained for N719-TBP was ascribed to a difference in molecular configuration. The molecular 
configuration of N719 has more isotropic character than N719-TBP which has the consequence that the direction of the emission dipole moment is allowed to be different from the direction of the absorption dipole moment, while in N719-TBP the absorption and emission dipoles tend to have the same direction. Thus one would expect a smaller depolarization of the fluorescent emission from N719-TBP than from N719, which by eq. (2) is equivalent to less fluorescence anisotropy in N719.

Figure 6 shows the polarization fluorescence anisotropy obtained for the DSC samples $D S C_{100 / 0}$ (solid, red), $D S C_{50 / 50}$ (solid, green) and $D S C_{0 / 100}$ (solid, blue) as a function of wave number. For comparison the re-measured $r_{f}$ curves valid for the solutions of N719 (dashed, red) and N719-TBP (dashed, blue) are also shown. It follows from the figure that there is no difference in the polarized fluorescence anisotropy for DSCs containing either N719 or N719-TBP as opposed to the results found for the solutions of these. It follows that when either N719 or N719-TBP are chemisorbed to the $\mathrm{TiO}_{2}$ the polarization of the fluorescence is determined primarily by the orientation of the adsorbed molecules rather than by the relative small difference in the molecular configuration. The immediate conclusion is that we cannot use the polarization change of the fluorescence to judge the stability or aging of the DSC if the degradation product N719-TBP is also adsorbed to the $\mathrm{TiO}_{2}$ substrate. It is noticed, however, that the $r_{f}$ values for the N719-TBP in solution are significantly larger than the corresponding values for the DSCs where $r_{f} \approx 3$ and that the $r_{f}$ values for N719 in solution are significantly smaller than for the DSCs. This seems to be an important result: If e.g. a number of N719 molecules become desorbed under the aging process the polarization fluorescence anisotropy will decrease, whereas when a number of molecules N719TBP become desorbed the polarization fluorescence anisotropy will increase. Before discussing this further the polarized RRS spectra are considered.

Since, as already discussed, the Raman bands above $1700 \mathrm{~cm}^{-1}$ are weak and the prominent bands below $600 \mathrm{~cm}^{-1}$ are mainly due to the Raman scattering in the $\mathrm{TiO}_{2}$ substratethe discussion of the polarized RRS is focused on the spectral region between 650 and $1700 \mathrm{~cm}^{-1}$.

We have obtained polarized RRS spectra in this spectral region for the DSC samples: $D S C_{100 / 0}$, $D S C_{80 / 20}, \quad D S C_{50 / 50}, \quad D S C_{20 / 80}$, and $D S C_{0 / 100}$. As representative examples the fluorescence corrected

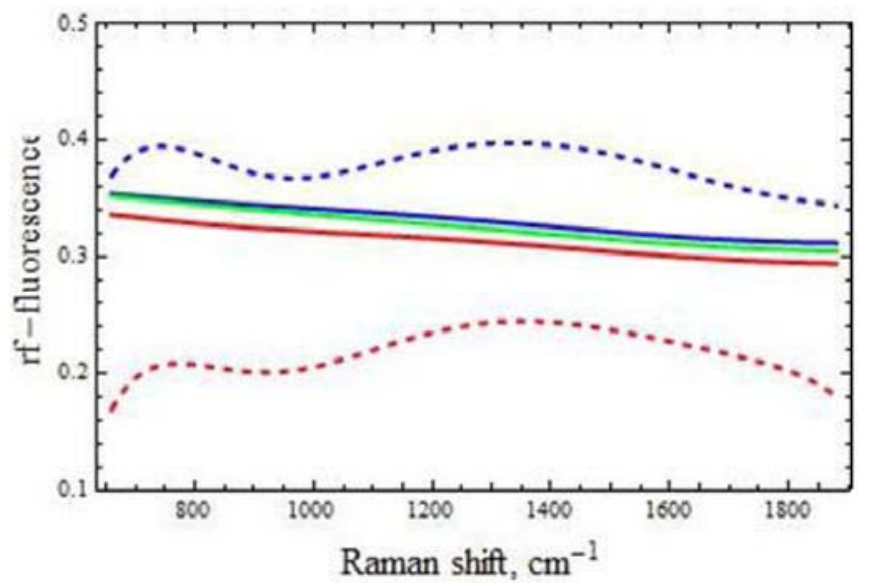

Figure 6: The polarization fluorescence anisotropy $r_{f}$, $D S C_{100 / 0}$ (solid, red), $D S C_{50 / 50}$ (solid, green) and $D S C_{0 / 100}$ (solid, blue) as a function of wave number. For comparison the re-measured $r_{f}$ for the solutions of N719 (dashed, red) and N719-TBP (dashed, blue) are also shown. Estimated uncertainty \pm 0.035 .

spectra for $D S C_{100 / 0}$ and $D S C_{0 / 100}$ is shown in Figures 7 and 8 . The black and green curves correspond to the perpendicularly polarized spectra $\left\langle I_{1,3, p e r}\right\rangle$ and parallel polarized spectra $I_{2, p a r}$, respectively. The spectra of $\left\langle I_{2,4, p a r}\right\rangle$ and $I_{3, \text { per }}$ are not shown, since they are similar, the only difference is that the signal to noise ratio is smaller. For comparison purposes the Raman bands have been scaled relative to the intensity of the parallel polarized component of the strongest Raman band at $1536 \mathrm{~cm}^{-1}$ (band 9).

The DPR values for the samples $D S C_{100 / 0}$ and $D S C_{0 / 100}$ for the bands $1-10$ in Figures 7 and 8 are calculated by processing the polarized data as previously explained and applying eq. (1). The DPR values are presented in Table $\mathbf{1}$ together with the band positions obtained from the fitting the un-polarized and polarized Raman data.

For comparison the DPR values for the samples $D S C_{100 / 0}$ and $D S C_{0 / 100}$ and the uncertainties are shown in Figure 9 together with the DPR values calculated for the re-measured solutions of N719 and N719-TBP. The full red and blue curves are for the samples $D S C_{100 / 0}$ and $D S C_{0 / 100}$ respectively, while the dashed red and dashed blue curves are for the solutions of N719 and N719-TBP.

First, a comparison of the DPR values for the samples $D S C_{100 / 0}$ and $D S C_{0 / 100}$ in Figure 9 shows that they will be different for the bands $3,4,7,8,9$ and 10 , while for the band 1, 2 and 5 no difference is observed. The latter result differs from the results found for these 


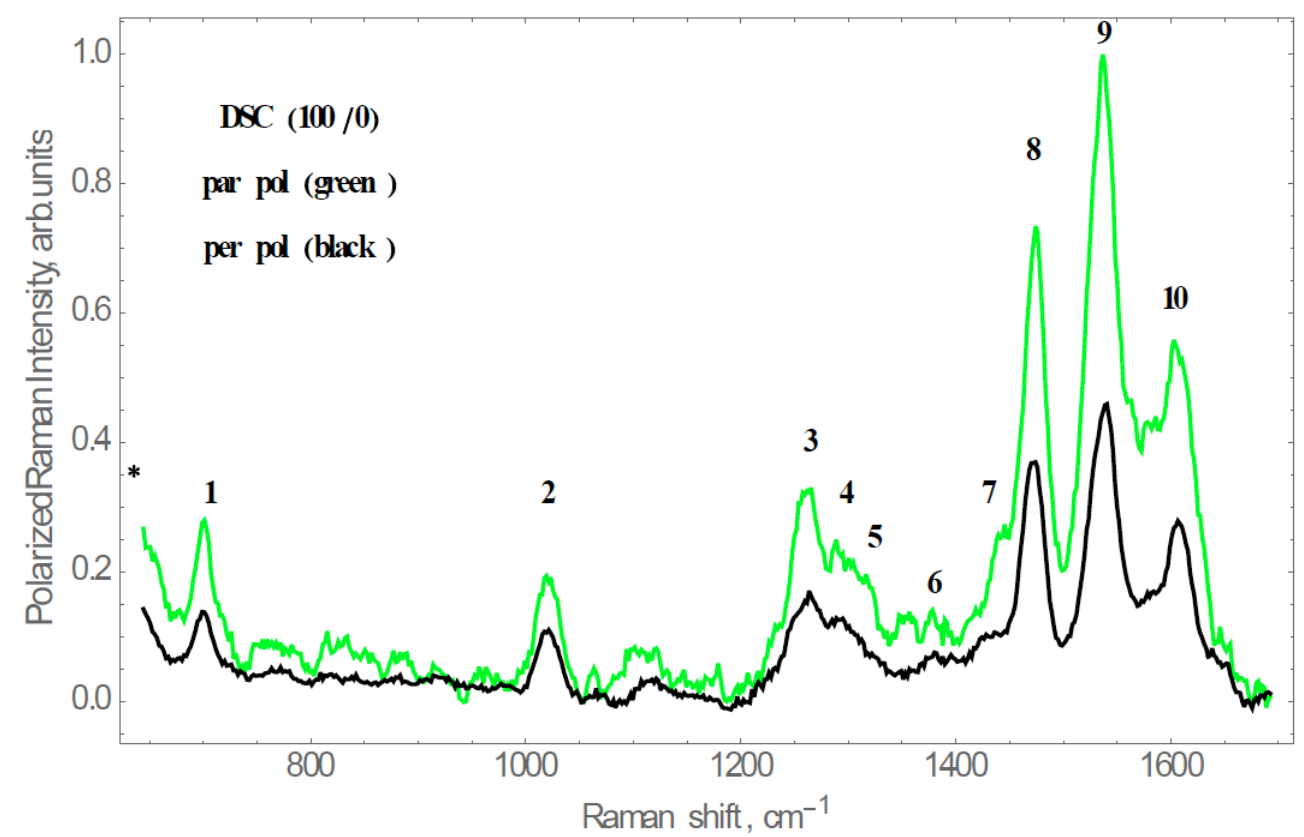

Figure 7: Fluorescence corrected polarized resonance Raman spectra of the sample $D S C_{100 / 0}$, parallel polarized (green) and perpendicularly polarized (black). The spectra are scaled relative to band 9 at $1536 \mathrm{~cm}^{-1}$ in the parallel polarized spectrum. Raman bands are marked $1-10$. * is a $\mathrm{TiO}_{2}$ band.

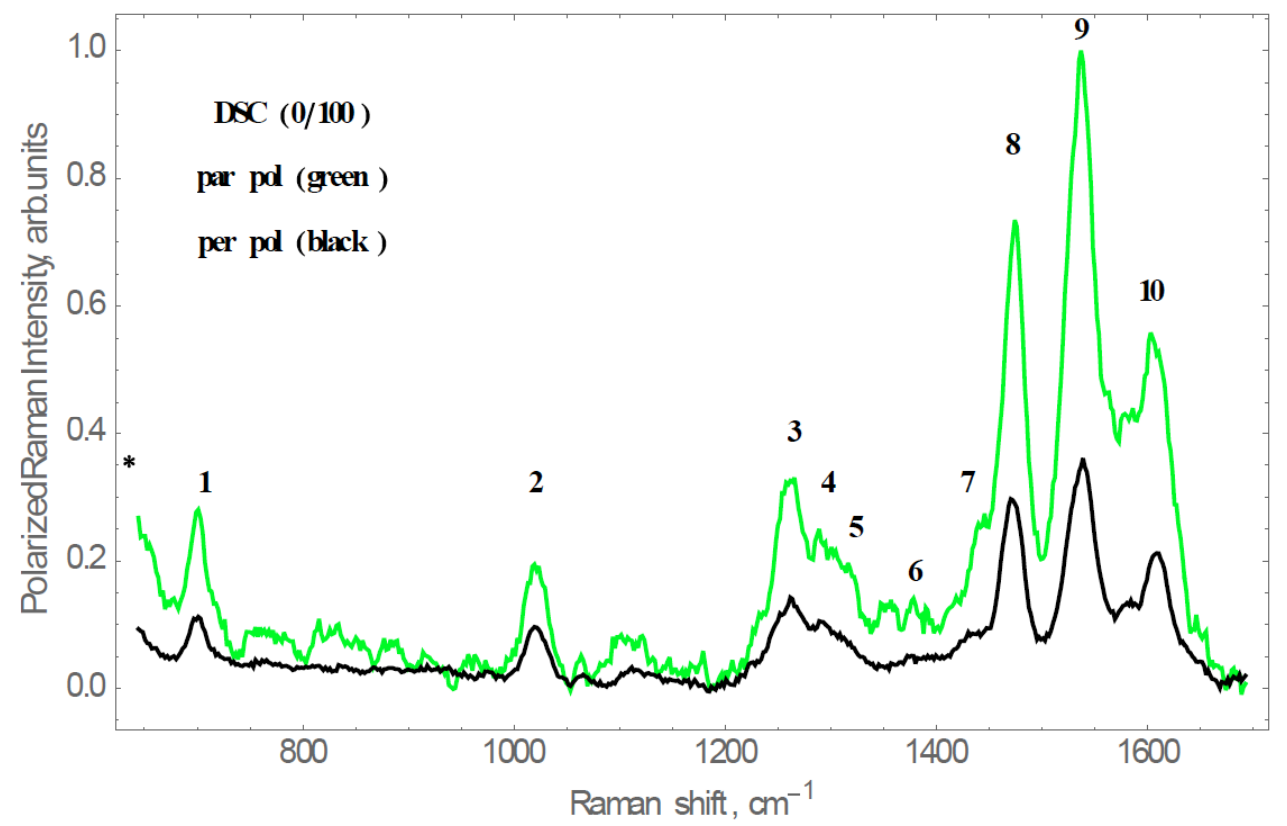

Figure 8: Fluorescence corrected polarized resonance Raman spectra of the sample $D S C_{0 / 100}$, parallel polarized (green) and perpendicularly polarized (black). The spectra are scaled relative to band 9 at $1536 \mathrm{~cm}^{-1}$ in the parallel polarized spectrum. Raman bands are marked $1-10$. ${ }^{*}$ is a $\mathrm{TiO}_{2}$ band.

bands in the corresponding solutions. Secondly, it follows from Figure 9 that the largest difference between the DPR values occurs between the values obtained for the DSCs and the values for the corresponding dye in solution (see below).

The origin of the observed differences in the DPR values found between the samples $D S C_{100 / 0}$ and
$D S C_{0 / 100}$ between the dye solutions of $\mathrm{N} 719$ and N719TBP and between e.g. $D S C_{100 / 0}$ and N719 in solution (i.e. between adsorbed and desorbed dye molecules) is as follows: For molecules with low or no symmetry all components in the Raman tensor will in general be different from zero. One consequence is that the DPR in general depends on the excitation wave number (polarization dispersion), when the excitation is applied 
Table 1: DPR Values for $D S C_{100 / 0}$ and $D S C_{0 / 100}$ Obtained from an Optimized Fit of the Polarized Resonance Raman Data as Described in the Subsection Signal Processing. Band Positions are Readout from Spectrometer and Band Positions Obtained from Fit with Gaussian Line Shape are Given in ( )

\begin{tabular}{|c|c|c|c|c|}
\hline Band & $\begin{array}{c}\text { Band } \max . / \mathrm{cm}^{-1} \\
D S C_{100 / 0}\end{array}$ & $\begin{array}{c}\text { DPR } \\
D S C_{100 / 0}\end{array}$ & $\begin{array}{c}\text { Band max. } / \mathrm{cm}^{-1} \\
D S C_{0 / 100}\end{array}$ & $\begin{array}{c}\text { DPR } \\
D S C_{0 / 100}\end{array}$ \\
\hline 1 & $701(700)$ & 0.59 & $701(700)$ & 0.59 \\
\hline 2 & 1023 (1022) & 0.55 & $1021(1022)$ & 0.55 \\
\hline 3 & 1264 (1262) & 0.54 & $1262(1261)$ & 0.49 \\
\hline 4 & 1287 (1290) & 0.72 & 1289 (1289) & 0.64 \\
\hline 5 & $1312(1313)$ & 0.53 & $1312(1316)$ & 0.52 \\
\hline 6 & 1378 & - & 1378 & - \\
\hline 7 & 1445 (1442) & 0.33 & 1445 (1442) & 0.22 \\
\hline 8 & $1474(1473)$ & 0.50 & $1472(1473)$ & 0.43 \\
\hline 9 & $1536(1538)$ & 0.42 & $1536(1537)$ & 0.37 \\
\hline 10 & 1608 (1608) & 0.44 & 1603 (1608) & 0.38 \\
\hline
\end{tabular}

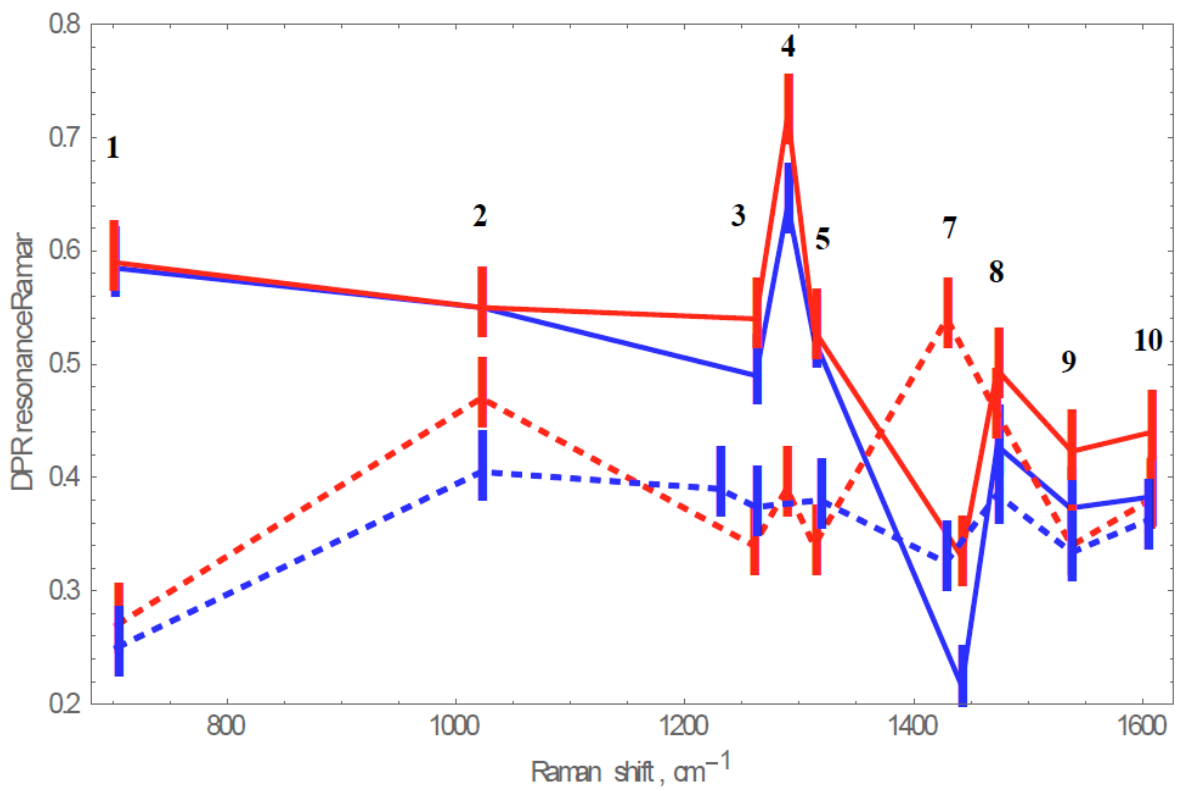

Figure 9: DPR values and estimated uncertainty \pm 0.025 for the $D S C$ samples: $D S C_{100 / 0}$ (solid, red) and $D S C_{0 / 100}$ (solid, blue), for the solutions of N719 (dashed, red) and N719-TBP (dashed, blue). The DPR values for the DSCs are calculated from data processing of the polarized spectra in Figure 7 and 8 and the DPR for the solutions from the re-measured spectra of the dye solutions in [15].

close resonance with an electronic transition. Raman modes where the DPR shows dispersion are called dispersive. If only a single excitation wave number is applied, as it is the case here, the DPR values for dispersive Raman modes will then depend on the wave number difference between the wave number of the laser and the wave number of the electronic transition responsible for the visible absorption spectrum.

The measured visible absorption spectra for the DSC samples $D S C_{100 / 0}$ and $D S C_{0 / 100}$ are shown in
Figure 10 together with the absorption spectra obtained for the dyes in solution. From these spectra it follows that the absorption spectrum of the $D S C_{0 / 100}$ (solid blue curve) is blue shifted approximately $30 \mathrm{~nm}$ relative to the absorption spectrum of the $D S C_{100 / 0}$ (solid red curve), while the absorption spectrum of the N719-TBP solution (dashed blue curve) is only blue shifted approximately $13 \mathrm{~nm}$ relative to the absorption spectrum of the N719 solution (dashed red curve). Finally the absorption spectrum of $D S C_{100 / 0}$ is red shifted approximately $24 \mathrm{~nm}$ relative to the absorption 
spectrum of the N719 solution. Thus the energy of the excited electronic state of N719 responsible for the visible absorption is decreased, when the dye molecule is chemisorbed to the $\mathrm{TiO}_{2}$ substrate as one would expect. It follows that these wave number shifts in the absorption spectra combined with the dispersive property of the Raman modes will give rise to changes in the DPR values, where the magnitude and sign of the DPR shifts will depend on the dispersion curve for the individual Raman modes and on the particular wave number of the laser used for the excitation. It follows from Figure 8 that the DPR graphs for $D S C_{100 / 0}$ (solid red) and N719 (dashed red) are similar, but that the average change of the DPR is large. Comparison of the graphs for $D S C_{0 / 100}$ (solid blue) and N7619-TBP (dashed blue) gives a similar result.

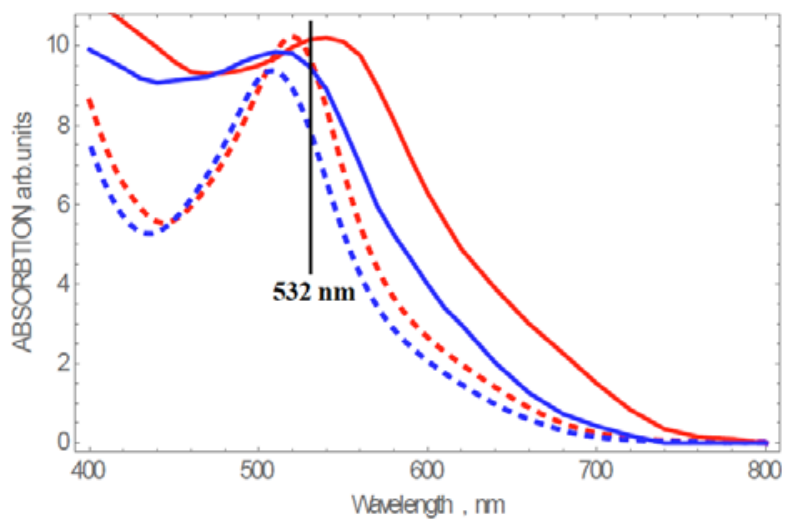

Figure 10: Comparison of the visible absorption spectra for $D S C_{100 / 0}$ (solid red), $D S C_{0 / 100}$ (solid blue), N719 in methanol (dashed red) and N719-TBP in methanol (dashed blue). The wavelength of the laser applied in the RRS experiments is marked with a vertical bar. For comparison the absorption spectra have been scaled relative to the maximum of the $D S C_{100 / 0}$ spectrum.

The large change in the DPR values observed between adsorbed and non-adsorbed dye molecules may be explained by the fact that while the nonadsorbed molecules are randomly oriented the molecules adsorbed to the $\mathrm{TiO}_{2}$ substrate are partially oriented. The scattering conditions for the Raman process are therefore very different in the two situations. In the case of Raman scattering from an oriented molecule the DPR is determined by the ratio between the absolute square of those components of the Raman tensor, which are selected by the molecular orientation in the laboratory system (defined by the laser polarization and the polarization analyzers). For randomly oriented molecules (solution and powders) the DPR values are determined by the rotational invariants of the Raman tensor. The expression for the DPR and the relations between the invariants and the components of the Raman tensor can be found in [14].
When the DPR values for the adsorbed dye molecules are compared with the DPR of the non-adsorbed molecules, it follows that the change of the DPR must reflect that both the molecular orientation and the wave number of the absorption spectra are changed. The large average increase of the DPR values, observed for the DSC relative to the N719 in solution, is probably due to the partial orientation exhibited by the N719 molecules, when they are adsorbed to the $\mathrm{TiO}_{2}$. The similarity between the DPR-graphs for the DSCs and the solutions shows that these changes are essentially due to the shifts in the absorption spectra combined with the dispersive property of the individual Raman modes. Since we observe very small wave number shifts in the Raman spectra for the resonance enhanced Raman modes the nature of these are therefore similar for adsorbed and desorbed molecules.

The average orientation of the adsorbed molecules depends on the details in the adsorption and the spatial structure of the $\mathrm{TiO}_{2}$ substrate. Since for oriented molecules the DPR is determined essentially by the ratio between the absolute square of those components of the Raman tensor, which are selected by the molecular orientation, more information about the adsorption might be extracted from an extended analysis (involving more experiments to be performed) of polarized RRS measurements.

\section{CONCLUDING REMARKS}

Raman spectroscopy is an attractive experimental technique for on-site investigation of dye sensitized solar cells. It is a fast, non-destructive molecule specific technique, which requires no special sample preparation and the excitation and collection of the Raman scattered light can be performed through the cover glass of the DSCs. However, as can be seen from the cited literature and which also follows from the discussion given above, the spectral changes in the unpolarized Raman and resonance Raman spectra that are generated by a degradation of the DSC or may arise when the ratio between adsorbed and desorbed dye molecules is changed, are generally small and unfortunately associated with the Raman modes with lowest intensity and overlapping Raman bands. These observations in combination with the fact that a relative strong fluorescence is generated simultaneously with the resonance Raman process have the consequence that the signal processing becomes crucial with respect to the accuracy of the analysis of the bands with low $\mathrm{S} / \mathrm{N}$ ratio. We have demonstrated that by analyzing the polarization changes in both the resonance Raman 
spectra as well as in the fluorescence instead of focusing only on the (un-polarized) Raman spectra themselves, it will be possible to greatly increase the contents of information which can be extracted from the data and since the polarization changes are now encountered in the strongest Raman bands the accuracy and the reliability of the results can be increased. Finally due to the application of the CCDtechnique in Raman spectrometers it is possible to modify the optics in a Raman microscope in such a way that both polarizations of the Raman scattered light can be measured simultaneously. This has two important consequences: the general $\mathrm{S} / \mathrm{N}$ ratio can be increased considerably by obtaining spectra in different points of the sample and average, but most important it opens the possibility of performing polarized resolved Raman and resonance Raman imaging on the DSCs. Work on this is in progress.

\section{REFERENCES}

[1] Oregan B, Grätzel M. A low-cost, high-efficiency solar cell based on dye-sensitized colloidal TiO2 films. Nature 1991; 353: 737-40. http://dx.doi.org/10.1038/353737a0

[2] Grâtzel M. Solar energy conversion by dye-sensitized photovoltaic cells. Inorg Chem 2005; 44: 6841-51. http://dx.doi.org/10.1021/ic0508371

[3] Grätzel M. Recent advances in sensitized mesoscopic solar cells. Acc Chem Res 2009; 42: 1788-1798. http://dx.doi.org/10.1021/ar900141y

[4] Hagfeldt A, Boschloo G, Sun L, Kloo L, Pettersson H. Dyesensitized solar cells. Chem Rev 2010; 110: 6595-663. http://dx.doi.org/10.1021/cr900356p

[5] Likodimos V, Stergiopoulos T, Falaras P, Hariksun R, Desilvestro J, Tulloch, G. Prolonged light and thermal stress effects on industrial dye-sensitized solar cells: a MicroRaman investigation on the long-term stability of aged cells. J Phys Chem C 2009; 113: 9412-22. http://dx.doi.org/10.1021/jp901185f

[6] Kontos A G, Stergiopoulos T, Likodimos V, et al. Long-term thermal stability of liquid dye solar cells. J Phys Chem C 2013; 117: 8636-46.

http://dx.doi.org/10.1021/jp400060d

[7] Greijer H, Lindgren J, Hagfeldt A. resonance Raman scattering of a dye-sensitized solar cell: mechanism of thiocyanato ligand exchange. J Phys Chem B 2001; 105: 6314-20. http://dx.doi.org/10.1021/jp011062u

[8] Hugot-Le Goff A, Falaras P. Origin of new bands in Raman of dye monolayers adsorbed on nanocrystalline $\mathrm{TiO}_{2}$. J Electrochem Soc 1995; 142: L38-41. http://dx.doi.org/10.1149/1.2048578
[9]

Peres Leon C, Kador L, Peng B, ThelakkatM. Characterization of the adsorption of Ru-bpy dyes on mesoporous TiO2 films with UV-Vis, Raman and FTIR spectroscopies. J Chem Phys B 2006; 110: 87234-30.

[10] Kato N, Takeda $\mathrm{Y}$, Higuchi K, et al. Degradation analysis of dye-sensitized solar cell module after long-term stability test under outdoor working condition. Solar Energy Mater Solar Cells 2009; 93: 893-7. http://dx.doi.org/10.1016/i.solmat.2008.10.022

[11] Agresti A, Pescetelli S, Quatela A, et al. Micro-Raman analysis of reverse bias stressed dye-sensitized solar cells. RSC Adv 2014; 4: 12366- 75. http://dx.doi.org/10.1039/c3ra47797e

[12] Hirose F, Kuribayashi K, Shikaku M, Narita Y. In situ observation of N719 on TiO2 in dye-sensitized solar cell by IR absorption spectroscopy. Electrochem Solid-State Lett 2009; 12: B167-70. http://dx.doi.org/10.1149/1.3238474

[13] Nazeeruddin MK, Humphry-Baker R, Liska P, Grätzel M. Investigation of sensitizer adsorption and the influence of protons on current and voltage of a dye-sensitized nanocrystalline $\mathrm{TiO}_{2}$ solar cell. J Phys Chem B 2003; 107: 8981-7.

http://dx.doi.org/10.1021/jp022656f

[14] Mortensen OS, Hassing S. Polarization and interference phenomenain resonance Raman scattering. In: Clark $\mathrm{R} \mathrm{J} \mathrm{H}$, Hester R E, editors. Advances in Infrared and Raman Spectroscopy Vol. 6, Heyden and Sons, New York 1980; p. $1-60$.

[15] Hassing S, Jernshøj K D, Nguyen PT, Lund T. Investigation of the stability of ruthenium-based dye (N719) utilizing the polarization properties of dispersive Raman modes and/or of the fluorescent emission. J Phys Chem C 2013; 117: 235006. http://dx.doi.org/10.1021/jp406596p

[16] Andersen AR, Halme J, Lund T, et al. Charge transport and photocurrent generation characteristics in dye solar cells containing thermally degraded N719 dye molecules. J Phys Chem C 2011; 115: 15598-606.

http://dx.doi.org/10.1021/jp201658

[17] Nguyen TP, Lam BTX, Andersen AR, Hansen PE, Lund T. Photovoltaic performance and characteristics of dye sensitized solar cells prepared with the N719 thermal degradation products $\left[\mathrm{Ru}(\mathrm{L}-\mathrm{H})_{2}(\mathrm{NCS})(4\right.$-tert-butylpyridine $\left.)\right]$, ${ }^{+} \mathrm{N}(\mathrm{Bu})_{4} \quad$ and $\quad\left[\mathrm{Ru}(\mathrm{L}-\mathrm{H})_{2}(\mathrm{NCS})(1-\right.$ methylbenzimidazole $\left.)\right]$, ${ }^{+} \mathrm{N}(\mathrm{Bu})_{4}$. Eur J Inorg Chem 2011; 16: 2533-9. http://dx.doi.org/10.1002/ejic.201000935

[18] Nguyen HT, Ta HM, Lund T. Thermal thiocyanate ligand substitution kineticsof the solar cell dye N719 by acetonitrile, 3-methoxypropionitrile, and 4-tert-butylpyridine. Solar Energy Mater Solar Cells 2007; 91: 1934-42. http://dx.doi.org/10.1016/j.solmat.2007.07.011

[19] Lakowicz JR. Principles of Fluorescence Spectroscopy. 2006; Springer. http://dx.doi.org/10.1016/j.solmat.2007.07.011

[20] Zhang Z, Chen S, Liang Y, et al. An intelligent backgroundcorrection algorithm for highly fluorescent samples in Raman spectroscopy. J Raman Spec 2009; 41: 659-69. http://dx.doi.org/10.1002/jrs.2500

\section{DOI: http://dx.doi.org/10.6000/1929-6002.2016.05.01.4}

(c) 2016 Hassing et al.; Licensee Lifescience Global.

This is an open access article licensed under the terms of the Creative Commons Attribution Non-Commercial License (http://creativecommons.org/licenses/by-nc/3.0/) which permits unrestricted, non-commercial use, distribution and reproduction in any medium, provided the work is properly cited. 